\title{
Two-Dimensional Elastic Bandgap Crystal to Attenuate Surface Waves
}

\author{
F. Meseguer, M. Holgado, D. Caballero, N. Benaches, C. López, J. Sánchez-Dehesa, and J. Llinares
}

\begin{abstract}
Experiments on the attenuation of Rayleigh waves in a marble quarry by a periodic array of cylindrical holes, in a honeycomb and in a triangular distribution, are reported. The results are explained in terms of elastic wave crystals (EWC's), and compared to theoretical calculations performed with scalar waves. The scaling property of the underlying theory has led us to explore the possible application of the results obtained to the attenuation of surface waves in seismic movements.
\end{abstract}

Index Terms -Elastic bandgap crystal, Rayleigh waves, surface waves.

\section{INTRODUCTION}

$\mathbf{S}$ INCE discovering photonic crystals (PC's) a decade ago, Yablonovitch [1] and John [2] have awakened an enormous interest [3]. The theory of PC can be extended to other classical waves, such as sound or elastic waves. In general, these materials can be called wave crystals (WC's), and can be these either PC's, sonic crystals (SC's), or elastic wave crystals (EWC's). The parameters that control the appearance of full bandgaps are varied:

1) the type of symmetry of the structure;

2) the wave velocity contrast;

3) the filling factor, defined as the ratio between the volume occupied by each dielectric with respect to the total volume of the composite;

4) the topology, which can be either cermet, where scattering centers (i.e., low-velocity material isolated from each other) or network, (scattering centers are connected); and the shape of the scattering centers.

All these factors determine the band structure of the WC and, therefore, wave propagation properties. Economou and Sigalas have published a general discussion about topologies in PC theory and the generalization to other classical waves [4]. Also, it has been shown that a slight modification of either the symmetry [5], [6] or the shape of the scatterer [7] can enlarge the gap.

Recently, there has been increasing interest in SC and EWC composites because of their application in sound filters and in

Manuscript received March 22, 1999; revised August 13, 1999. This work was supported in part by the Comisión Interministerial de Ciencia y Tecnología of Spain under Contract MAT97-0698-C04, Generalitat Valenciana, and Consejo Superior de Investigaciones Científicas.

F. Meseguer, M. Holgado, N. Benaches, C. López, and J. Llinares are with Unidad Asociada CSIC-UPV, Edificio de Institutos II, Valencia 46022 Spain. They are also with Instituto de Ciencia de Materiales (CSIC), Cantoblanco, Madrid 28049 Spain.

D. Caballero and J. Sánchez-Dehesa are with the Departamento de Física Teórica de la Materia Condensada (UAM) Cantoblanco, Madrid 28049 Spain. Publisher Item Identifier S 0733-8724(99)08850-7. vibrationless environments. Most of the work concerns theoretical calculations [8]-[10], and very few concern experiments [11]-[13]. Also, some of us have shown that, under certain conditions, minimalist sculptures show full gaps in the audible region of the EM spectrum. Also, the existence of the so-called "deaf bands" can increase the effective bandgap as obtained in SC experiments [14]. Recently, Tanaka and Tamura have presented calculations of the dispersion relations of surface phonons in a two-dimensional (2-D) AlAs-GaAs composite structure [15].

One important consequence of the WC theory is the scalability of the equations that governs the propagation of the waves. Any wave phenomena that appears for a certain range of wavelengths and length scale can be extrapolated to other systems whose order length is scaled up or down with respect the previous one; i.e., if we halve the length scale we double the energies.

Surface effects are very important in elastic wave propagation, where strong coupling effects to bulk waves occur. This is of paramount importance in geophysics because Rayleigh and Love waves are highly destructive. To the best of our knowledge, neither theory nor experiments concerning the attenuation of surface waves by an EWC have been reported.

We have studied the attenuation of Rayleigh waves by a 2-D periodic distribution of cylindrical holes distributed in the surface of a marble quarry. The motivation of this work comes from the possibility of applying the concept of EWC to attenuate surface seismic waves.

\section{EXPERIMENT}

We have performed the experiments in a marble quarry for several reasons.

1) A quarry is a suitable place to produce surface waves because it presents wide areas where the surface is very flat.

2) The marble thickness underneath the surface is much larger than the penetration length of surface waves.

3) It constitutes a system similar to the real system we would like to represent, the surface of the earth.

4) In our opinion, it constitutes a closer approach than a laboratory experiment. Also, the realization of a similar experiment in a laboratory would require large blocks of materials that are very difficult to handle and with undesirable wave reflection at the borders.

In general, quarries present faults, breaches, and cracks that distort wave propagation. Therefore, prior to performing any experiment, it was necessary to test many quarries and 


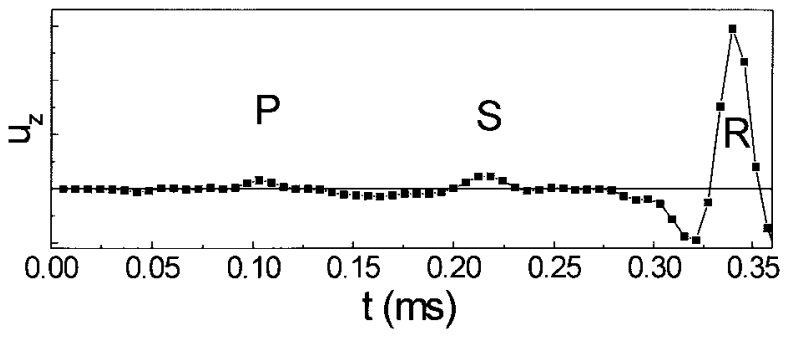

Fig. 1. Typical seismogram produced by the impact of the bearing ball. The arrival of the primary (P) or longitudinal and secondary (S) or transversal waves can be observed, before the Rayleigh wave (R) reaches the detector.

many places in the selected quarry to decide the best zone. The suitable place was decided upon by taking into account the following aspects: 1) the wave should be isotropically distributed, and 2) the contamination of the wave transmission from spurious reflections should be as small as possible.

Surface elastic waves are generated by the impact of a 0.5 -in steel bearing ball. This specific size has been chosen because it produces an easily detectable signal as well as a short impact. A white spectrum elastic wave $\left(0<\omega<\omega_{0}=40 \mathrm{kHz}\right)$ is generated. Brüel and Kjaer piezoelectric detectors with a flat spectral response in the frequency range $\left(0<\omega<\omega_{0}=20\right.$ $\mathrm{kHz})$ are used. Out of this range, the signal is not reliable as resonances in the detector itself disturb the measurement. In all cases, we have measured the vertical polarization of the surface wave; i.e., we detect the vertical component of the polarization of the waves perpendicular to quarry surface. The amplified signal from detectors is fed to a data acquisition card in a personal computer. This method allows us to obtain time domain data, and select the time domain window to avoid spurious reflections. Fig. 1 shows a typical time domain wave where longitudinal $(\mathrm{P})$, transversal $(\mathrm{S})$, and Rayleigh (R) waves can be seen. From that, a value for the Rayleigh wave velocity $c_{R}=2960 \pm 30 \mathrm{~ms}^{-1}$ has been obtained. After, to obtain a frequency domain spectrum, a Fast Fourier Transform analysis of data is performed.

The 2-D EWC samples under study are constructed by the following procedure. In a first stage, we bored 85 holes in a honeycomb lattice (3-m point group symmetry). After the attenuation experiments on this structure are performed, we drill 42 additional holes placed in the centers of the hexagons of the previous structure, so that it becomes a triangular lattice (6-mm point group symmetry) with a total number of 127 holes. The holes, $60 \mathrm{~mm}$ in diameter, are bored with a drilling machine intended to take marble probes, being the center to center distance between the closest holes $14 \mathrm{~cm}( \pm 3.5 \%)$. Fig. 2(a) and (b) shows a photograph of the quarry zone with the distribution of holes. As we progressed in drilling holes, we became aware of the existence of a sloping breach in the marble quarry at about $100 \mathrm{~cm}$ in depth at it shallowest point. This breach, which has a thickness of several centimeters, is filled with soil that strongly attenuates elastic waves, so we can consider that our crystal has an effective depth of $100 \mathrm{~cm}$.

The signal generated by the impact is measured simultaneously by two detectors. One is placed behind the holes array, so it feels the signal transmitted through the structure (sample detector). The second one (reference detector) is located at

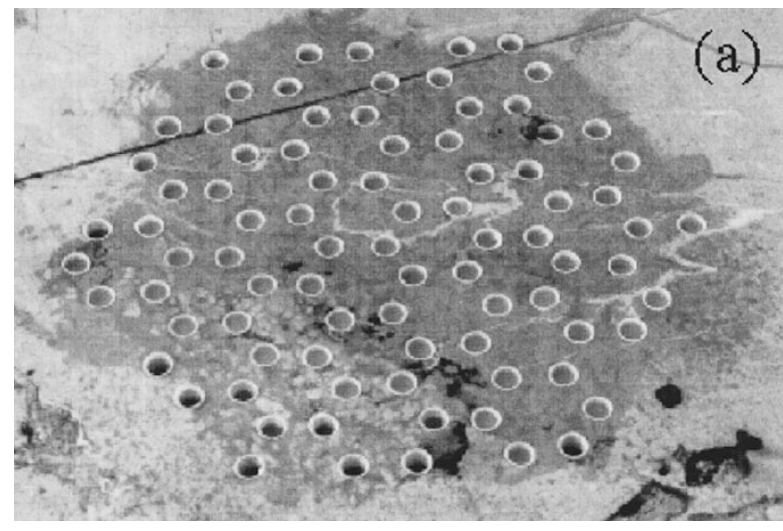

(a)

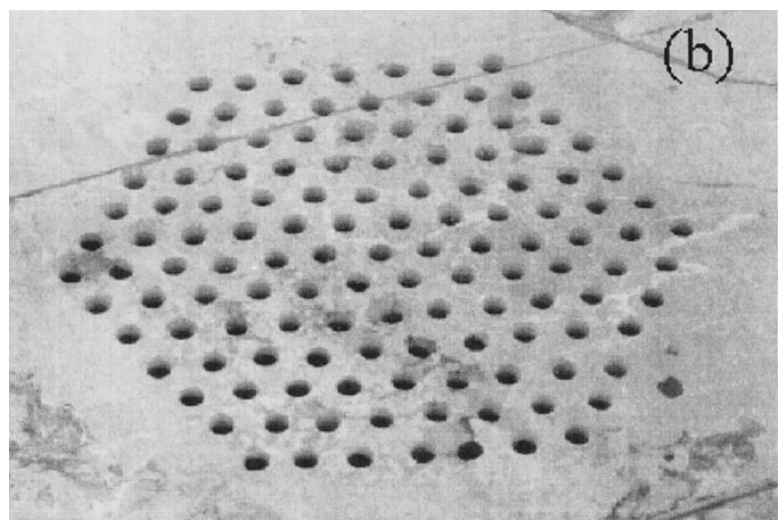

(b)

Fig. 2. Photographs of (a) the honeycomb and (b) the triangular arrangements of cylindrical holes drilled on the surface of the marble quarry. The external shape of the drilled zone forms a hexagon. The holes are $1.5 \mathrm{~m}$ in depth, and the distance between the closest ones is $14 \mathrm{~cm}$. The straight lines in the upper and the lower part of the structure correspond to shallow tracks (1 $\mathrm{cm}$ in depth) left by the saw machine in the process of extraction of the marble blocks. These tracks do not influence the experiments in the range of frequency values considered in this work.

the same distance from the exciting impact in a clean region of the quarry. To reach the latter, the signal does not cross the structure. The wave is generated far from the structure in order to insure that the waves are plane when they reach the scattering obstacle.

Figs. 3 and 4 show the wave attenuation (WA) spectra of the honeycomb and triangular structures for $k$-wavevectors along $\Gamma X$ and $\Gamma J$ directions of the Brillouin zone (BZ). Let us recall that the Bravais lattice of the honeycomb structure is 1.5 times larger that that of the triangular structure. The honeycomb structure has a smaller $\mathrm{BZ}$ and it is rotated $30^{\circ}$ with respect the triangular one. Therefore, $\Gamma X$ and $\Gamma J$ directions are interchanged in both symmetries (see insets). The negative values of WA are due to elastic wave reinforcements, probably due to increases in the signal arriving to the sample detector produced by inhomogeneities of the marble.

In systems like those here studied, as elastic waves find enormous density and velocity contrast, Bragg diffraction peaks (pseudogaps) could be transformed into very broad attenuation bands. Therefore, when broad attenuation bands appear, the concept of Bragg peak loses its sense. However, in order to have some guide, we have plotted as arrows the 


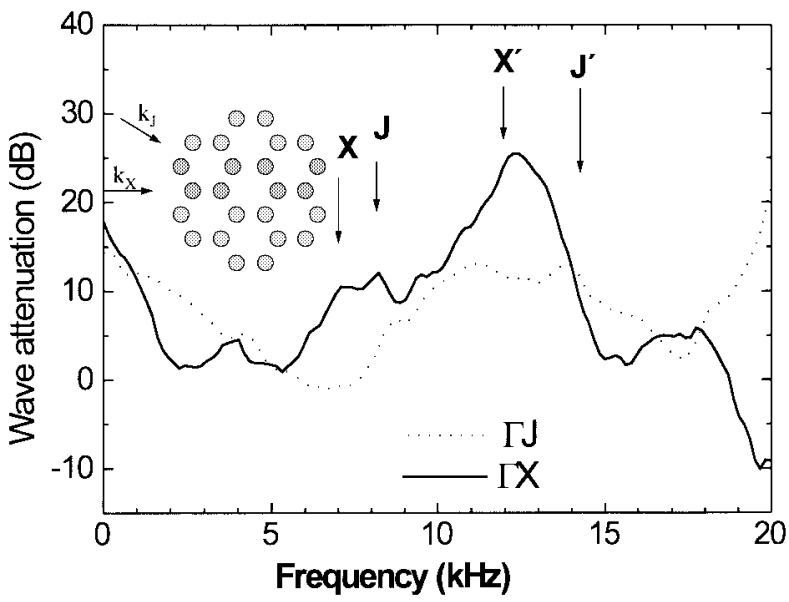

Fig. 3. Attenuation spectra of the honeycomb distribution of holes for directions of the wavevector along $X$ (solid curve) and $J$ (dotted curve) The arrows correspond to the Bragg frequencies values at $X, X^{\prime}, J$, and $J^{\prime}$ points of the BZ.

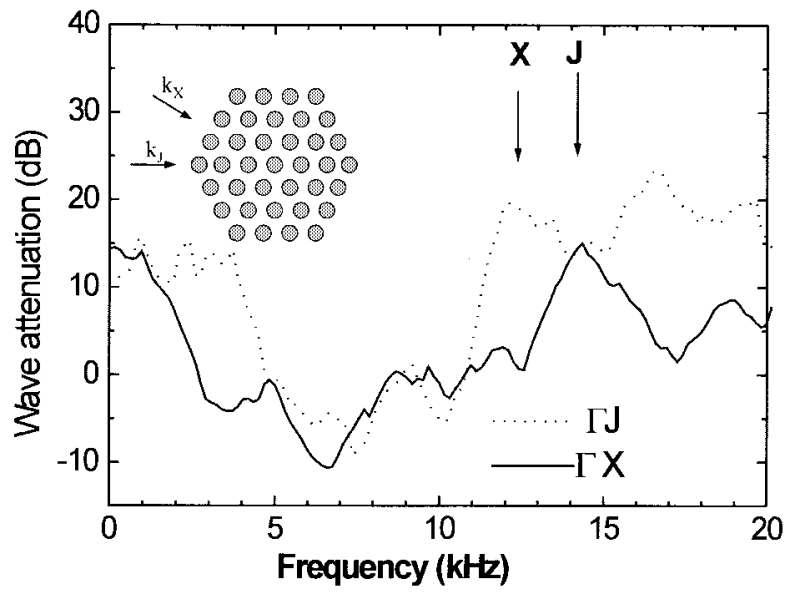

Fig. 4. Attenuation spectra of the triangular distribution of holes for directions of the wavevector along $X$ (solid curve) and $J$ (dotted curve). The arrows correspond to the Bragg frequencies values at $X$ and $J$ points of the $\mathrm{BZ}$.

frequency values estimated for the Bragg peaks at $X, X^{\prime}, J$, and $J^{\prime}$ points of the BZ.

The hexagonal structure (Fig. 3) shows two broad attenuation bands along $\Gamma X$ direction that cover a large part of spectral region of the detector for any direction of the $k$ wave vector. The first band is near zero frequency and it extends up to a cutoff frequency value of $2 \mathrm{kHz}$; the second one covers a frequency range between 7 and $15 \mathrm{kHz}$. Along $\Gamma J$ direction, a similar spectrum is obtained. However, a striking difference between $\Gamma X$ and $\Gamma J$ directions, other than the widths of the peaks, is the stronger attenuation observed along $\Gamma X$. We attribute this effect to the different number of layers that the Rayleigh wave has to cross before it reaches the detector (17 layers along $X$ and only 13 along $J$ ).

Fig. 4 shows the attenuation of Rayleigh waves through a triangular lattice for $k$ wavevectors along $X$ and $J$ directions of the BZ (see inset). Here again, two broad attenuation bands can be seen. The first one, as it occurs in Fig. 3, appears around a value of the frequency near zero. The second band goes from $11 \mathrm{kHz}$ and goes beyond frequency value that is above the frequency limit of the detector $(20 \mathrm{kHz})$. In comparison with the one of the honeycomb structure, the second band is shifted to higher frequencies because the larger value of the reciprocal lattice. This band is stronger along the $\Gamma J$ direction (the direction equivalent to the $\Gamma X$ in the honeycomb) due to the different number of layers encountered by the wave. All the frequency values above mentioned have been assigned disregarding oscillations in the WA of $\pm 5 \mathrm{~dB}$ around the reference level. Finally, we have placed several sample detectors inside the triangular structure to study how spectra develops as wave crosses different numbers of layers along $\Gamma J$ direction. For nine monolayers, the attenuation spectrum is very similar to that obtained for the whole structure. Therefore, a few number of layers is enough to build up an EWC.

The experimental observations reported above cannot be accounted for by surface roughness effects [16], and, led us to conclude that two absolute elastic bandgaps exists for the propagation of Rayleigh waves. These gaps are defined by the overlap observed between the attenuation bands along the two high-symmetry directions.

We would like now to discuss whether those structures would show a full bandgap. There are several conditions that favor the full bandgap appearance [4], [8], [9]:

1) high symmetry;

2) cermet topology, i.e., the low-velocity scattering material (air) regions are isolated cylinders surrounded by the interconnected high-velocity material;

3) large values for the density and velocity contrasts.

All of these conditions are met in our experiment. We have a huge density contrast $\left(2.16 \times 10^{3}\right)$ and also a large velocity contrast (8.8) that would favor the full gap appearance. The high-frequency attenuation band for both triangular and honeycomb structures can be naively correlated to Bragg phenomena (see arrows in the figures). However, the lowfrequency band cannot be correlated to any Bragg diffraction peak. Attenuation bands in the low-frequency regime have also been characterized, both experimentally and theoretically, in the case of electromagnetic wave propagation through arrays of metal cylinders in air at the wavelenthgs, where the dielectric constant of the metal becomes infinite [17], [18]. In our case, due also to the huge density and velocity contrasts, strong band bending effects are taking place and a gap at near zero frequency is opened up. These results have important consequences because the structure is able to attenuate a broad range of waves whose wavelength is much larger than the coherence length (periodicity value) of the structure. In the next section, we will further comment on these effects in the light of theoretical calculations.

\section{THEORY AND DISCUSSION}

A theoretical description of the attenuation spectra implies the study of the scattering of Rayleigh waves by the array of finite air cylinders. Let $z=0$ be the stress-free surface of the semi-infinite medium occupying the half space $z \leq 0$. The components of the displacement field $u_{x}$ and $u_{z}$ of a 
Rayleigh wave propagating along the $x$-direction with a given $k$-wavenumber can be expressed as

$$
\begin{aligned}
u_{x}(x, z, t)= & A\left[e^{-k \beta_{L} z}-\left(1-\frac{c_{R}^{2}}{2 c_{T}^{2}}\right) e^{-k ; \beta_{T} z}\right] \\
& \cdot \cos k\left(x-c_{R} t\right) \\
u_{z}(x, z, t)= & A \beta_{L}\left[e^{-k \beta_{L} z}-\left(1-\frac{c_{R}^{2}}{2 c_{T}^{2}}\right)^{-1} e^{-k \beta_{T} z}\right] \\
& \cdot \sin k\left(x-c_{R} t\right)
\end{aligned}
$$

where $A$ is a constant, $c_{R}$ denotes the velocity of the Rayleigh wave, and $c_{T}$ and $c_{L}$ are the velocities of the transverse and longitudinal bulk elastic waves, respectively, while $\beta_{L}=$ $\sqrt{1-\left(c_{R}^{2} / c_{L}^{2}\right)}$ and $\beta_{T}=\sqrt{1-\left(c_{R}^{2} / c_{T}^{2}\right)}$. A calculation of the penetration depths $\left(k \beta_{L}\right)^{-1}$ and $\left(k \beta_{T}\right)^{-1}$ for the parameters of our system $\left(c_{L} \cong 6000 \mathrm{~ms}^{-1}\right.$ and $\left.c_{R} \cong 0.92 c_{T}\right)$ gives $\left(k \beta_{L}\right)^{-1}=0.18 \lambda$ and $\left(k \beta_{T}\right)^{-1}=0.38 \lambda$ [19]. Therefore, the depth of the drilled cylinder establishes a critical wavelength $\lambda_{c}$ such that any wave with smaller wavelength $\lambda$ sees the cylinders as infinitely long as far as the scattering process is concerned. In our structures $\lambda_{c}=4.2 \mathrm{~m}$. This implies that only those waves with frequency lower than $c_{R} / \lambda_{c} \cong 700$ $\mathrm{Hz}$ experience scattering in a finite depth structure. These waves are only a minor fraction of our spectra. The above discussion simplifies the theoretical attempt and one can consider the cylinders as semi-infinite for frequencies above $700 \mathrm{~Hz}$. This calculation is beyond the scope of the present work. Instead, we have employed a simple model, based on a scalar approach, that has given us good insight into the physics involved in our experiments. The model considers a periodic three-dimensional (3-D) system of cylindrical holes (scattering material, $s$ ) infinite along the $z$-axis, embedded in marble (host material, $h$ ). Thus, we calculate the band structure for the propagation of acoustic plane waves travelling through such a system. The corresponding equation is

$$
\nabla\left(\frac{\nabla p(r)}{\rho(r)}\right)=-\omega^{2} \frac{p(r)}{c^{2}(r) \rho(r)}
$$

where $p(r)=\rho(r) c^{2}(r) \nabla \cdot u(r)$ is the frequency of an eigenmode and $c(r)$ and $\rho(r)$ are the wave velocity and the medium density, respectively, which are position-dependent in the 2-D space $r=(x, y)$. As the wave velocity in the marble $c_{R}$, we have used the one measured for the Rayleigh wave.

We solve (3) using a variational method [14], [20]. Fig. 5(a) and (b) shows the dispersion relation, in reduced units, at very low values of the filling fraction $(0.5$ and $1 \%$ for the honeycomb and triangular lattices, respectively). In spite of the low ff, strong band bending effects occur in the honeycomb structure. This is because of the large density and velocity contrast of the marble air system. When we put the actual values of the $\mathrm{ff}$ used in our experiments ( $\mathrm{ff}=11.1 \%$ in the honeycomb case and $16.7 \%$ for the triangular one), the bands are extremely flat and highly degenerate (see Fig. 6). Particularly outstanding is that, in both structures, large gaps appear in the low-frequency region [notice the expanded scale in Fig. 6(a) and (b)]. This result obtained with our oversimplified model indicates that the intuitive picture of Bragg scattering fails to predict the position of attenuation bands in composite

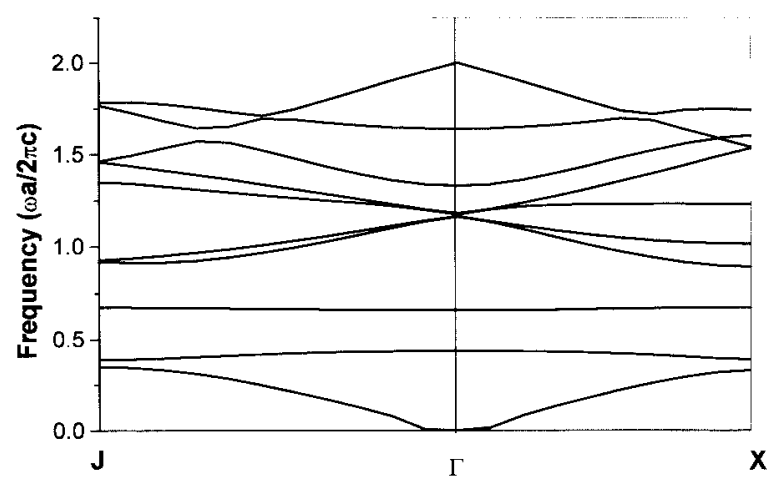

(a)

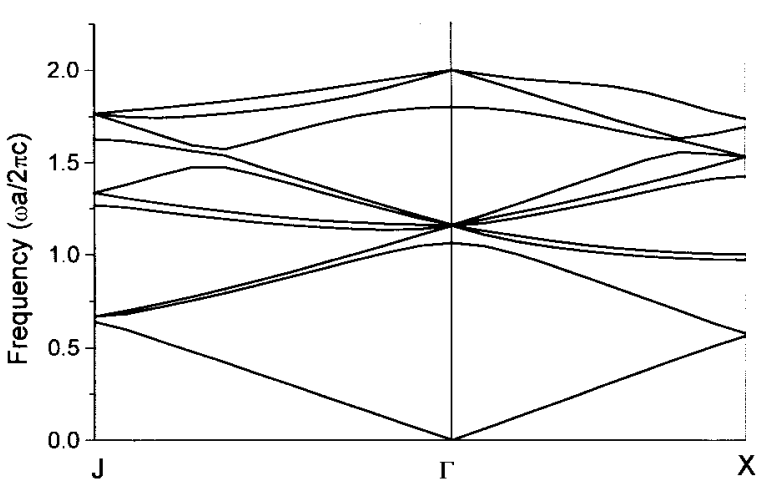

(b)

Fig. 5. Band structure, in reduced units $(\omega a / 2 \pi c)$ for scalar waves in a 2-D EWC for (a) the honeycomb and (b) the triangular symmetries and filling factor values of 0.5 and $1 \%$, respectively. For this calculation, we have used a wave velocity value for the Rayleigh wave, of $2960 \mathrm{~ms}^{-1}$ (see text).

systems with huge contrasts. It also supports our claim that the attenuation bands measured in the low-frequency region does represent actual gaps of the structure for the propagation of Rayleigh waves as it occurs in metallo-dielectric PBG structures [17]. However, the calculation does not reproduce either the direction dependence of the attenuation bands or the attenuation bands observed at high frequencies, probably due to the simplifications employed. Consequently, to account for the gaps found at high frequencies, a more realistic calculation is needed.

Because of the relevance of the low-frequency gap, we have also calculated the behavior of the quality factor, gap to midgap ratio $(\Delta \omega / \omega)$ associated with the first bandgap, as a function of the filling factor. The results obtained for honeycomb and triangular lattices are shown in Fig. 7. The parameter $(\Delta \omega / \omega)$ quickly saturates at very large values (around 1.8 in both cases), with the magnitude of the ff being very small ( 0.01 and 0.05 , respectively). Also, the calculation shows that the honeycomb structure is better than the triangular one, and indicates that very small values of the ff produce bandgaps with a very large value of the quality factor. This result is closely related to that obtained by other authors for 2-D [17] and 3-D [18] metallo-dielectric PBG structures.

Finally, it is very tempting to explore the possibility of application of these results to attenuate Rayleigh waves in earthquakes. The theory that governs the EWBG, and also the conclusion obtained from our experiments, are scaleable, i.e., 


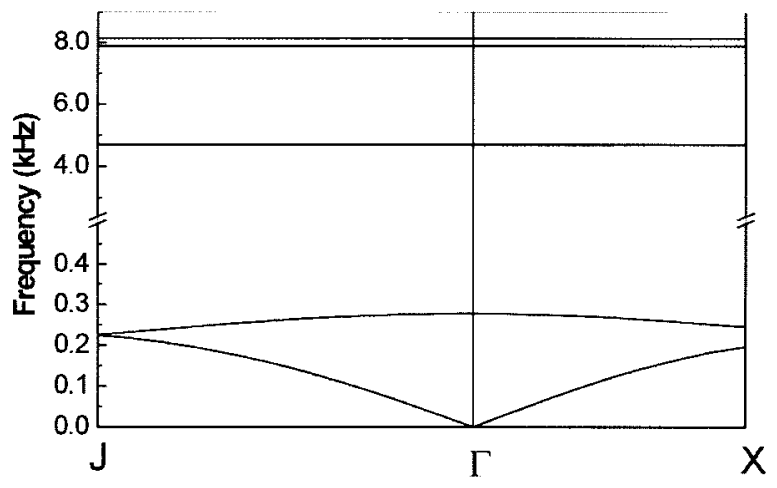

(a)

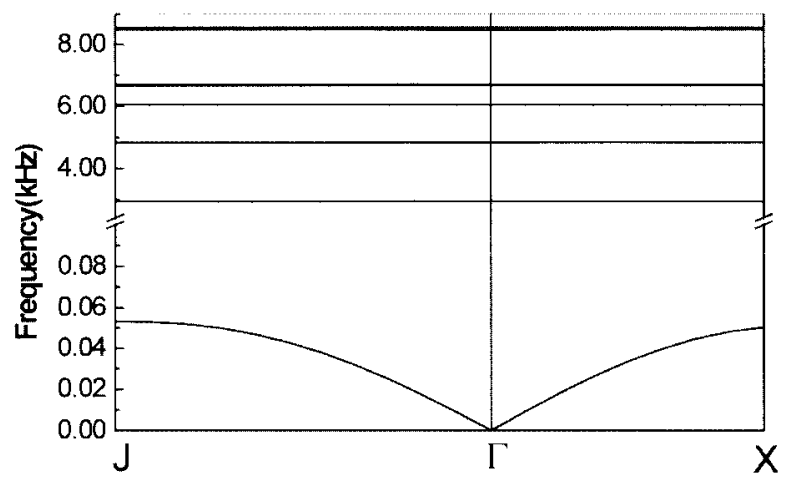

(b)

Fig. 6. (a) Band structure for scalar waves propagating in a 2-D EWC in a honeycomb distribution of infinite cylindrical holes in marble for a filling fraction of $11.1 \%$. (b) Same for triangular lattice with a ff of $16.7 \%$. In both calculations, we have considered $2960 \mathrm{~ms}^{-1}$ as the wave velocity in marble.

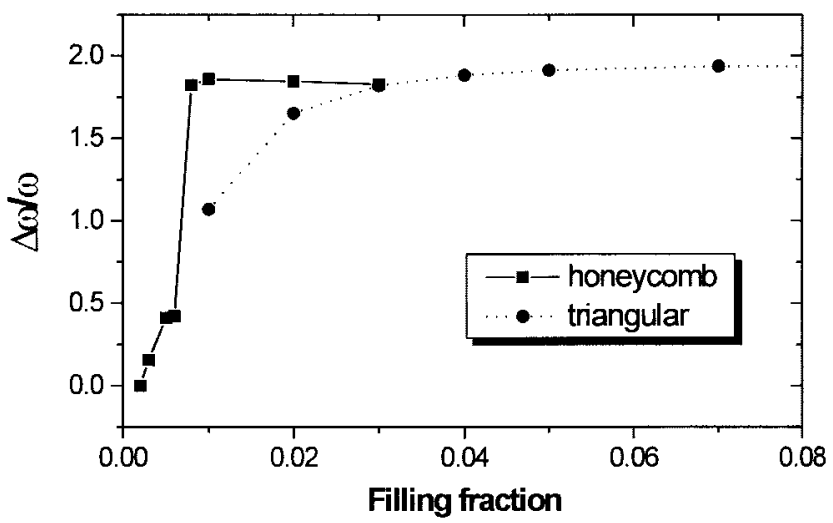

Fig. 7. Value of the bandgap width $\Delta \omega / \omega$ as a function of the filling factor for both honeycomb (full squares), and the triangular (full circles) symmetries.

any bandgap map of a wave crystal (WC) can be scaled up or down through the expansion or contraction of the length of the system. Seismic movements behave as elastic waves [21] with frequency values between $1 \mathrm{~s}^{-1}$ and $5 \mathrm{~s}^{-1}$. This corresponds to a bunch of waves whose bandwidth $(\Delta \omega)$ to mean frequency $(\varpi)$ ratio is $\Delta \omega / \varpi \approx 1.3$. From our calculations, this frequency range can be shielded by using the first gap for ff value around 1\%, in the honeycomb symmetry. This corresponds to a ratio cylinder diameterperiodicity $d / a=0.13$. If we assume that the velocity of the Rayleigh waves is of the order of $3000 \mathrm{~ms}^{-1}, 25 \mathrm{~m}$ holes periodically distributed in a honeycomb geometry, and $200 \mathrm{~m}$ apart would be needed. Also, due to the enormous contrasts both in velocity and density between the marble and the air, the cylindrical holes behave as hard cylinders, and elastic waves do not penetrate within the cylinder. One can take advantage of this fact and excavate circular trenches instead of digging up full cylindrical holes. It is much easier to perform cylindrical trenches (about $1 \mathrm{~m}$ wide) than emptying full cylindrical holes. This is of great importance as it makes the proposal more feasible for application in seismology. Notice that the hole depth has to be around $1200 \mathrm{~m}$ deep in order to shield waves of $1 \mathrm{~s}^{-1}$. A word of caution is required about the above proposal-the numbers are preliminary estimates and have to be checked with improved models and further experiments. For instance, it would be essential to study how deep the holes must be drilled in order to obtain acceptable earthquake attenuation effects. If holes are shallow in terms of the seismic wavelength, the surface wave would be diffracted due to edge effects, and it would be transmitted. Therefore, it is very important to study those factors that influence shielding effects of both frequency and intensity ranges that are dangerous for buildings and civil works [22].

\section{SUMMARY}

In summary, we have shown attenuation experiments of Rayleigh waves in a 2-D distribution of holes in a marble quarry. The results show the existence full gaps at low- and high-frequency regions. Theoretical calculations performed with scalar waves also predict large gaps in these type of systems. Because of the scaling property of the wave equations, it could be of application to attenuate the effects of Rayleigh waves in seismic movements. The gap obtained at near zero frequency allow us to make a proposal of seismic shielding based on an EWC structure of spatial periodicity much smaller than typical values of earthquake wavelengths. Further studies to know the feasibility of this application are dearly required.

\section{ACKNOWLEDGMENT}

The authors acknowledge J. Carrillo for the technical assistance in the drilling task, and H. Míguez, J. P. Hernandez, and F. J. García-Vidal for their critical reading of the manuscript.

\section{REFERENCES}

[1] E. Yablonovitch, "Inhibited spontaneous emission in solid state physics and electronics," Phys. Rev. Letters, vol. 58, p. 2059, 1987.

[2] S. John, "Strong localization of photons in certain disordered dielectric superlattices," Phys. Rev. Lett., vol. 58, p. 2486, 1987.

[3] "Science editors have presented PC as one of the six emerging fields for 1999," Science, vol. 282, p. 158, Dec. 18, 1998.

[4] E. N. Economou and M. M. Sigalas, "Classical wave propagation in periodic structures: Cermet versus network topology," Phys. Rev., vol. B48, p. 13434, 1993.

[5] D. Casagne, C. Jouanin, and D. Bertho, "New hexagonal structures for two-dimensional photonic band-gap materials," Nuovo Cimento, vol. D17, p. 1401, 1995.

[6] C. M. Anderson, and K. P. Giapis, "Large two-dimensional photonic band gaps," Phys. Rev. Lett., vol. 77, p. 2949, 1996.

[7] A. Chutinan and S. Noda, "Spiral 3 dimensional photonic band-gap structures," Phys. Rev., vol. B57, p. 2006, 1998.

[8] M. M. Sigalas and E. N. Economou, "Band structure of elastic waves in two dimensional systems," Solid State Commun., vol. 86, p. 141, 1993. 
[9] M. S. Kushwaha and P. Halevi, "Band gap engineering in periodic elastic composite," Appl. Phys. Lett., vol. 64, p. 1085, 1994.

[10] M. M. Sigalas and E. N. Economou, "Elastic waves in plates with periodic inclusions," J. Appl. Phys., vol. 75, p. 2845, 1994.

[11] L. Ye, G. Cody, M. Zhou, and P. Sheng, "Observation of bending wave localization and quasi-mobility edge in 2 dimensions," Phys. Rev. Lett., vol. 69, p. 3080, 1992.

[12] R. Martinez-Sala, J. Sancho, J. V. Sanchez, V. Gómez, J. Llinares, and F. Meseguer, "Sound attenuation by sculptures," Nature, vol. 378, p. $241,1995$.

[13] F. R. Montero de Espinosa, F. Jiménez, and M. Torres, "Ultrasonic band gap in a periodic two-dimensional composite," Phys. Rev. Lett., vol. 80, p. $1208,1998$.

[14] J. V. Sanchez, D. Caballero, R. Martinez-Sala, C. Rubio, J. SánchezDehesa, F. Meseguer, J. Llinares, and F. Gálvez, "Sound attenuation by two-dimensional array of rigid cylinders," Phys. Rev. Lett., vol. 80, p. 5325,1998

[15] Y. Tanaka and S. Tamura, "Surface acoustic-waves in 2-dimensional periodic structure," Phys. Rev., vol. B58, p. 7958, 1998.

[16] A. G. Eguiluz and A. Maradudin, Phys. Rev., vol. B28, p. 728, 1983

[17] D. R. Smith, S. Schultz, K. Kroll, M. Sigalas, K. M. Ho, and M. Soukoulis, "Experimental and theoretical results for a two-dimensional metal photonic band-gap cavity," Appl. Phys. Lett., vol. 65, p. 645, 1994.

[18] D. F. Sievenpiper, E. Yablonovitch, J. N. Winn, S. Fan, P. R. Villeneuve, and J. D. Joannopoulos, "3D metallo-dielectric photonic crystals with strong capacitive coupling between metallic islands," Phys. Rev. Lett., vol. 80, p. 2829, 1998.

[19] E. Soczkiewicz, "The penetration depth of Rayleigh surface waves," Acoustica, vol. 82, p. 380, 1986.

[20] F. Meseguer, M. Holgado, D. Caballero, N. Benaches, J. SánchezDehesa, C. López, and J. Llinares, Phys. Rev., vol. B59, p. 12169 , 1999.

[21] K. Aki and P. C. Richards, Quantitative Seismology. San Francisco, CA: Freeman, 1980

[22] K. Kanai, Engineering Seismology.Tokyo, Japan: Tokyo Univ. Press 1983.
F. Meseguer, photograph and biography not available at the time of publication.

M. Holgado, photograph and biography not available at the time of publication.

D. Caballero, photograph and biography not available at the time of publication.

N. Benaches, photograph and biography not available at the time of publication.

C. López, photograph and biography not available at the time of publication.

J. Sánchez-Dehesa, photograph and biography not available at the time of publication.

J. Llinares, photograph and biography not available at the time of publication. 